(1973)

15) Prausnitz, J. M. and F. H. Shair: AIChE J., 7, 682 (1961).

16) Puri, P. S., J. Polak and J. A. Ruether: J. Chem. Eng. Data, 19, 87 (1974).

17) Riddick, J. A. and W. B. Bunger: "Organic Solvents", 3rd ed., Wiley-Interscience, New York (1970).

18) Röck, H. and W. Schröder: Z. Physik. Chem. (Frankfurt), 11, 41 (1957).
19) Tasić, A., B. Djordjević, D. Grozdanić, N. Afgan and D Malić: Chem. Eng. Sci., 33, 189 (1978).

20) Tokunaga, J.: J. Chem. Eng. Japan, 8, 7 (1975).

21) Wilhelm, E. and R. Battino: J. Chem. Thermodynamics, 5, 117 (1973).

22) Van Ness, H. C. and M. M. Abbott: Ind. Eng. Chem., Fundam., 17, 66 (1978).

\title{
WAKE BEHAVIOR AND ITS EFFECT ON INTERACTION BETWEEN SPHERICAL-CAP BUBBLES
}

\author{
ISAO KOMASAWA, TSUTAO OTAKE \\ AND MoToYoshi KAMOJIMA \\ Department of Chemical Engineering, Osaka University, \\ Toyonaka, Osaka 560
}

\begin{abstract}
The dynamical behavior of a single and a pair of spherical-cap bubbles was investigated while they were held stationary by means of a downward liquid flow in a vertical channel. No significant differences were found in behavior such as bubble geometry, rising velocity, or liquid velocity profile around a bubble, for free-rising bubbles in a stationary liquid pool and for the present bubbles held stationary in a water channel.

The fluid dynamical properties of the wake behind spherical-cap bubbles were classified by Reynolds number, as a laminar wake $(10<\operatorname{Re}<90)$, a transitional wake $(90<\operatorname{Re}<500)$, and a turbulent wake $(R e>500)$. The laminar wakes of the preceding bubbles had a decisive effect on the rising velocity of the trailing bubbles, in which an additional velocity component caused by the wakes of the preceding bubbles was correlated well with the wake velocities. The turbulent wakes had a limited effect on the acceleration of the trailing bubbles and often caused them to break up.
\end{abstract}

\section{Introduction}

During the free-rise of gas bubbles through a liquid an amount of liquid is inevitably carried up behind the bubbles, and this is known as the wake. In the last decade it has been realized that the wakes play a significant role in the interaction between bubbles. Several reports have been published in this field. Crabtree and Bridgwater ${ }^{2}$ applied, for the whole range of a wake, a wake velocity function which is probably valid only far downstream from the bubble. De Nevers and $\mathrm{Wu}^{4}$ ) used an ideal wake model, in which the wake had the same velocity as that of a bubble. Narayanan et al. ${ }^{7}$ ) presented an empirical correlation for the approach velocities of two bubbles. For a swarm of bubbles, Otake et al.$^{8}$ found a complicated interaction process of the bubbles caused by the wakes.

Received August 8, 1979. Correspondence concerning this article should be addressed to I. Komasawa. M. Kamojima is now with Toyo Eng., Co., Funabashi 273.
When free-rising gas bubbles are brought to rest with respect to an observer, the behavior of a bubble or a pair of bubbles can be observed for a prolonged length of time. This method therefore appears to be very useful for the examination of the dynamical properties of bubbles, and especially the interaction between bubbles. It is, however, necessary to examine beforehand whether there might be some differences in the geometry and dynamical properties between free-rising bubbles in a stationary infinite liquid pool and bubbles held stationary in a liquid stream.

In the present work, the geometry and rising velocity for single spherical-cap bubbles held stationary were first determined and compared with the literature data for single bubbles rising freely in an infinite liquid pool. The wake structure and the liquid velocity of the wake were investigated in an attempt to evaluate the momentum content of the wake, since these were supposed to have a direct effect on the behavior of neighboring bubbles. The interaction of the two bub- 


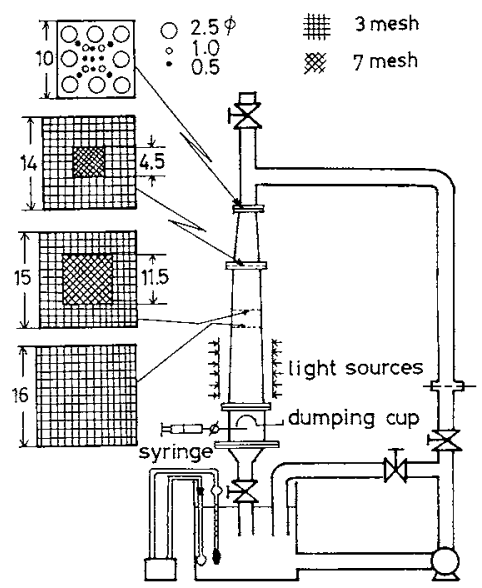

Fig. 1 Schematic diagram of counter-current flow apparatus

Table 1 The properties of liquids (water jelly solutions)

\begin{tabular}{ccccccc}
$\begin{array}{c}C \\
{[\mathrm{wt} \%]}\end{array}$ & $\begin{array}{c}\mu \\
{[\mathrm{CP}]}\end{array}$ & $\begin{array}{c}T \\
{\left[{ }^{\circ} \mathrm{C}\right]}\end{array}$ & $\begin{array}{c}\rho \\
{\left[\mathrm{g} / \mathrm{cm}^{3}\right]}\end{array}$ & $\begin{array}{c}\sigma \\
{[\mathrm{dyne} /} \\
\mathrm{cm}]\end{array}$ & $\begin{array}{c}M \\
{[-]}\end{array}$ & $\begin{array}{c}\text { Keys in } \\
\text { Fig. 3 }\end{array}$ \\
\hline 81.2 & 711.7 & 24.0 & 1.33 & 78.7 & 3.9 & $\bullet$ \\
78.0 & 335.6 & 22.0 & 1.32 & 77.4 & & $\emptyset$ \\
74.2 & 183.8 & 24.0 & 1.31 & 76.7 & $1.9 \times 10^{-2}$ & $\oplus$ \\
70.0 & 135.6 & 18.0 & 1.28 & 76.5 & & $\oplus$ \\
64.9 & 63.0 & 18.0 & 1.27 & 76.8 & $1.1 \times 10^{-3}$ & $\Theta$ \\
58.1 & 34.2 & 18.0 & 1.25 & 75.6 & & $\bigcirc$ \\
50.0 & 13.5 & 19.0 & 1.19 & 75.1 & $2.6 \times 10^{-4}$ & $\triangle$ \\
30.0 & 3.2 & 20.0 & 1.11 & 74.6 & & $\nabla$ \\
0 & 1.0 & 20.0 & 1.0 & 73.8 & $2.4 \times 10^{-11}$ & $\square$
\end{tabular}

bles caused by the wakes was studied in detail and finally an empirical correlation was developed for the acceleration effects of the wakes on the neighboring bubble.

\section{Experiment}

The free-rising bubbles were made stationary with respect to an observer by means of a downward liquid flow field in a vertical flow channel. The schematic diagram of the apparatus is shown in Fig. 1. The test section of the channel was made of a Perspex "pyramidal frustum section", $70 \mathrm{~cm}$ high with a 14$\mathrm{cm}$ square at the top and a $17-\mathrm{cm}$ square at the base. In an attempt to obtain a flat velocity profile at the test section, the use of several mesh screens, funnels, and some spoiler systems in the channel were investigated. The three mesh screens and a perforated plate were finally found to produce a flat velocity profile with a decayed turbulence at the central core part of the test section. The assembled unit shown in Fig. 1 was capable of holding gas bubbles $0.8 \mathrm{~cm}$ to $3.7 \mathrm{~cm}$ in equivalent diameter for at least 5 minutes.

A predetermined volume of air was injected into the column by means of a hypodermic syringe and stored under the cup. The bubble was released by inverting the cup and held stationary at an observation level in the test section by adjusting the liquid velocity. The liquid velocities and streamlines were measured by means of solid tracer particles suspended in the liquid. Intense plane-collimated beams of light from two $1-\mathrm{kW}$ Xe-arc lamps were used to illuminate a vertical $0.7 \mathrm{~cm}$ wide slice of the column. The suspended particles illuminated by the beam were photographed with a Nikon camera equipped with a micro-Nikkor lens and a motordrive attachment. The liquid velocities and stream lines were determined from the trajectories of the particles in an exposure time of $1 / 8$ or $1 / 15 \mathrm{sec}$. The rising velocities of the bubbles were equivalent to the liquid velocities when the bubbles were held stationary. The tracer particles used were polystyrenedivinylbenzene copolymer particles of 50-mesh size with a density of $1.05 \mathrm{~g} / \mathrm{cm}^{3}$.

A pair of bubbles rising in line was obtained by releasing the second bubble, after the first bubble had been held stationary at the upper part of the test section of the column. The interaction of the two bubbles was recorded with a Milliken cine-camera, and the position-time behavior of the two bubbles was determined by measuring the positions on the film with a NAC motion analyser.

Deionized water and water-jelly (malt honey) solutions $(0-82 \mathrm{wt} \%)$ were used. The viscosities were measured with a viscometer (Visconic END, Tokyo Keiki Co. Ltd.) and the interfacial tensions by a pendant-drop method. The properties of liquids are given in Table 1.

\section{Results and Discussion}

\subsection{Behavior of single bubbles}

From the data of the basal diameter $d_{b}$ and the height $h$, the parameters of the spherical-cap bubbles were calculated using the following correlations ${ }^{3,11)}$ :

$$
\begin{aligned}
& \theta_{m}=180-2 \tanh \left(d_{b} / 2 h\right) \\
& R=\left(d_{b} / 2\right) / \sin \theta_{m} \\
& V_{b}=\left(\pi R^{3} / 3\right)\left(2-3 \cos \theta_{m}+\cos ^{3} \theta_{m}\right) \\
& d_{e}=\left(6 V_{b} / \pi\right)^{1 / 3}
\end{aligned}
$$

1) Liquid velocity profile around a bubble

The liquid velocity profile at several horizontal planes, both ahead of and behind the bubble, were determined at low Reynolds number. The profile at a plane through the center of the vortex ring is plotted in Fig. 2, and compared with the profile determined experimentally for a free-rising bubble in a stationary glycerol solution at the same Reynolds number ${ }^{12)}$ and also with that predicted by Parlange based on Hill's vortex theory ${ }^{9}$. The Parlange model failed to describe the present result. The present result, however, is in good agreement with that for a free-rising bubble ${ }^{12}$. The velocity profile immediately ahead of the bubble is found to be well described by a potential flow analy- 


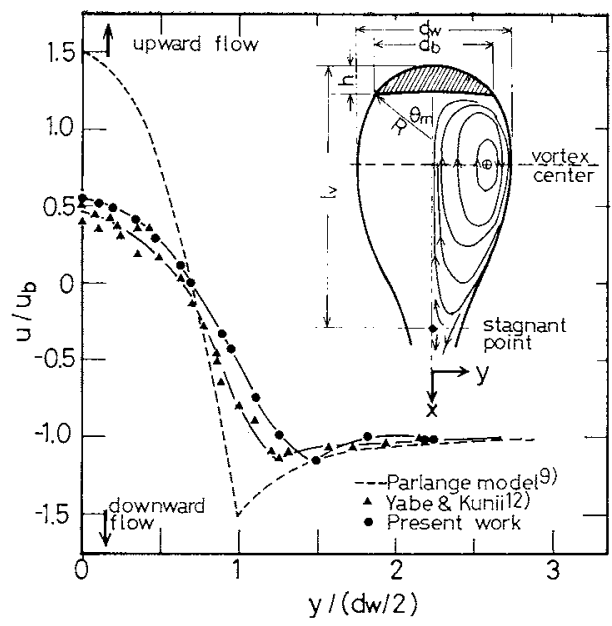

Eig. 2 Velocity profile at a plane through the center of vortex rings at $R e=84$

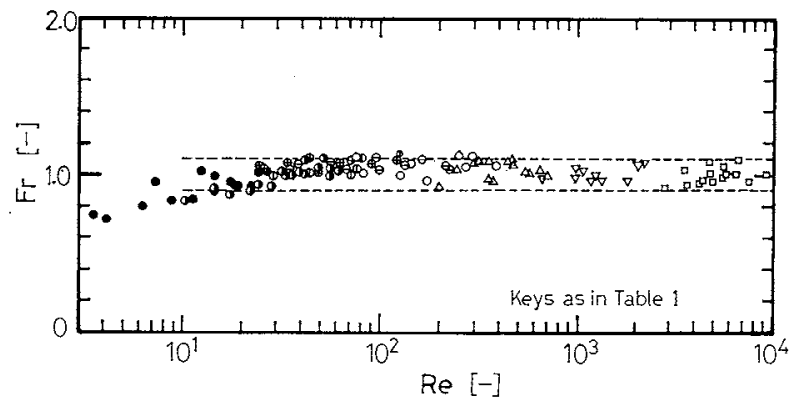

Fig. 3 Rising velocity of spherical-cap bubbles

sis, as was found for the free-rising bubble ${ }^{12}$.

2) Rising velocity

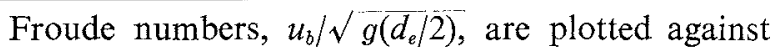
the corresponding bubble Reynolds numbers in Fig. 3. All the data fall within the range $0.9<F r<1.1$. The present results agree well with the data collected by Wegener and Parlange ${ }^{11}$ for free-rising bubbles in stationary liquid pools, and they also agree well with the theoretical value of 1.0 presented by Davies and Taylor ${ }^{3}$.

In water and liquids with viscosity less than $5 \mathrm{cP}$, the shape of the bubbles becomes irregular and fluctuating, and the volume $V_{b}$ was therefore determined from the volume of air injected into the column. For these bubbles Froude numbers are the same as those for the stable spherical-cap bubbles as shown in Fig. 3.

\section{3) Geometry of bubbles}

For the ratio of the equivalent diameter to the basal diameter, $d_{e} / d_{b}$, Tadaki and Maeda ${ }^{10)}$ presented a constant value of 0.62 in the range of $R e \cdot M^{0.23}>16.5$. Davies and Taylor ${ }^{3)}$ presented a value of 0.57 . The present data obtained in the range $13<R e \cdot M^{0.25}<60$ are found to fall between the two predictions obtained for free-rising spherical-cap bubbles in a stationary liquid pool.
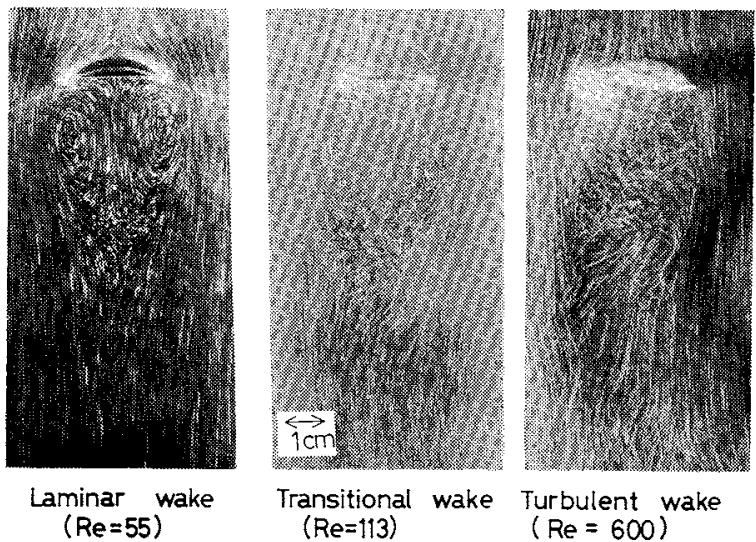

Fig. 4 Typical photographs of wakes

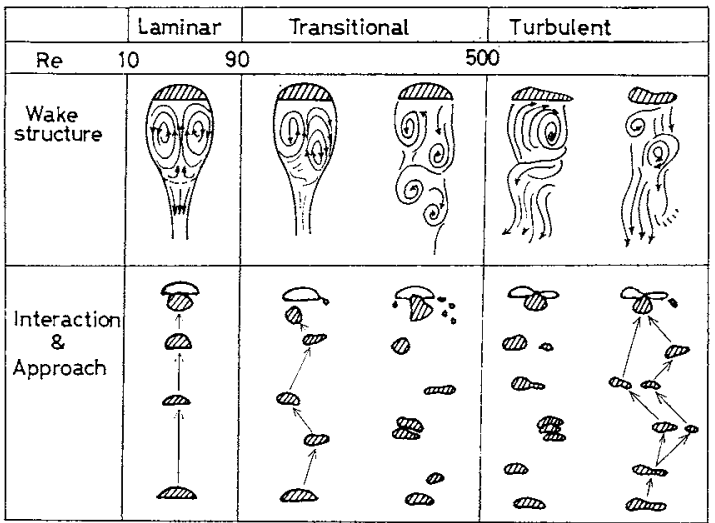

Fig. 5 Sketches of wake behavior and interaction of two bubbles

Consequently, no significant differences have been found in behavior such as bubble geometry, rising velocity, and liquid velocity profile around a bubble, for free-rising bubbles in a stationary liquid pool and for the present bubbles held stationary in a water channel. This has also been partially confirmed by Coppus et al. ${ }^{1)}$ and Kojima et al. ${ }^{5}$. Thus the present bubble system is considered to be acceptable for the study of wakes and their effect on the interaction of bubbles.

\section{2 Wake behavior}

1) General observation of wakes

Typical wake behavior is illustrated by the photographs in Fig. 4 and by sketches in Fig. 5. The behavior of the wakes depends on Reynolds number, which is determined mainly by viscosities in the present experiment. The following three typical types of wake were observed.

(i) Laminar wake In the range of $10<R e<90$, the wake is stable and consists of a toroidal vortex and a streaming cylindrical tail extending along the rise path. The toroidal vortex contains a pair of symmetrical oblate vortices. Between the toroidal vortex and the cylindrical tail, there is a point where the liquid is apparently stagnant. This is depicted as 


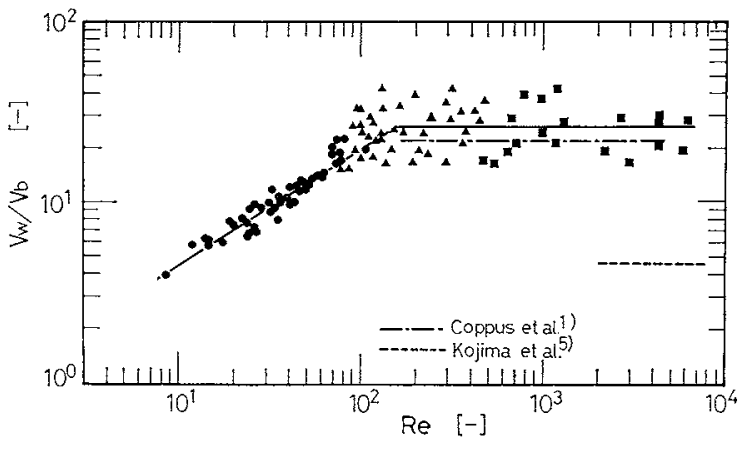

Fig. 6 Wake volume of spherical-cap bubbles

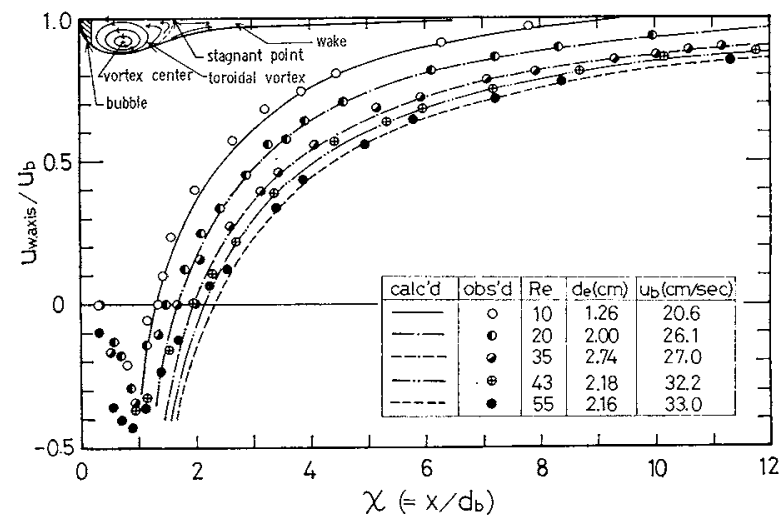

Fig. 7 Velocity distribution of liquid on symmetric axis of wake and comparison of observed and calculated values

a stagnant point in Fig. 2 .

(ii) Transitional wake In the range of $90<R e<250$, the wake becomes less stable, i.e., the oblate vortices in the toroidal vortex becomes less symmetrical and a rocking motion appears in the cylindrical tail. Although the stagnant point fluctuates mainly along the rise path, this point is still observed. On the whole, the wake still consists of a toroidal vortex and a cylindrical tail. At around Re: 300-500, a certain rolling motion appears on the toroidal vortex as well as on the cylindrical tail. The wake is elongated irregularly and shedding of the vortex ring is often seen.

(iii) Turbulent wake In the range of $R e>500$, small-scale irregular vortices are formed under the bubble and these shed alternatively. The streamlines of the wakes move side to side, and extend far behind the bubble. However, the path of the wake stream fluctuates and is rather difficult to follow on the film.

2) A measure of the intensity scale of wakes

As a measure of the intensity of a wake, the size and velocity of the wake are determined. This is considered to be a clue to how the bubble interaction is effected.

(i) Wake volume Even for a laminar wake with a stable, clear boundary, the cylindrical tail narrows continuously. As a measure of the wake volume, the following volume is defined for the laminar and transitional wake $(\operatorname{Re}<200)$.

$$
V_{w}=\left(\pi d_{w}^{2} / 4\right)\left(l_{v}-h\right)
$$

where $d_{w}$ is the maximum diameter of the wake and $\left(l_{v}-h\right)$ is the distance between the rear surface of the bubble and the stagnant point of the wake. For a turbulent wake, the length is defined, following Kojima et al. ${ }^{5}$, as the distance between the rear surface of the bubble and the point in the wake where the streamlines of the wake (the trajectories on the film) intersect at right angles to the main flow.

The ratio of wake volume to bubble volume is plotted against Reynolds number in Fig. 6. In the range of $R e<200$, the ratio increases with Reynolds number and the empirical correlation is obtained as

$$
V_{w} / V_{b}=R e^{0.68}
$$

In the range of $R e>200$, the data are considerably scattered owing to difficulties in reading the trajectories on the film and to the fluctuating character of the wake. The ratios of wake to bubble volumes are independent of Reynolds number and have a mean value of 26 . This value is much greater than the value of $V_{w} / V_{b}=$ 4.7 found by Kojima et al.5). They used a small working section of $5 \mathrm{~cm}$ diameter, which might suppress wake behavior.

(ii) Wake velocity Except for a laminar wake, reproducible data would hardly be obtained owing to the fluctuating character of the wake. The actual laminar wake has a velocity gradient in both directions, radial and longitudinal (along the rise path). As an index of wake velocity, liquid velocities on the symmetric axis of the laminar wake were determined. The results are plotted in Fig. 7, where $u_{b}$ is the rising velocity of a bubble (or the velocity of liquid flowing apart from the bubble), and $\chi\left(=x / d_{b}\right)$ is the dimensionless distance from the front surface of the bubble. The tail of a wake was found to extend a long distance downstream from a bubble. The ratio, $u_{w, \text { axis }} / u_{b}$, decreases with distance, and at $\chi=1.2-2.0$ it becomes zero. This point is a stagnant point, at which the liquid velocity is the same as that of the bubble. In the region of toroidal vortex, the value of the ratio becomes less than zero, since the liquid motion on the axis is upward. At $\chi=0.8-1.0$, the value falls to minimum. This means that the liquid velocity of the upward motion becomes greatest at a horizontal plane through the vortex center.

The wake velocities can be expressed as a function of Reynolds number and the distance as*

* Two different expressions in the wake should have been made, for the toroidal vortex region $(0<\chi<1.2-2.0)$ and for the cylindrical tail region $\left(1.2-2.0<\chi<\chi_{c}\right)$. As an approximation, Eqs. (7) and (9) are adopted as representative in the range $1<\chi<\chi_{c}$. 


$$
\begin{aligned}
& u_{w, \text { axis }} / u_{b}=f(\operatorname{Re}, \chi) \text { for } 1<\chi<\chi_{c} \\
& u_{w, \text { axis }} / u_{b}=1 \quad \text { for } \chi>\chi_{c}
\end{aligned}
$$

where $\chi_{0}$ is a critical separation distance. The results are correlated as follows:

$$
u_{w, \mathrm{axis}} / u_{b}=1.3 R e^{-0.05}-0.85 R e^{0.27} / \chi
$$

The calculated curves according to the above empirical equation are also shown in Fig. 7. Some deviations are seen in the range $\chi=1-2$, especially for high Reynolds number.

\section{3 Interaction of bubbles}

1) General observation of the behavior of a pair of bubbles

A series of photographs of two approaching bubbles with laminar wakes is illustrated in Fig. 8. For bubbles with laminar wakes, the following direct observations were made.

(i) The trailing bubble has no effect on velocity or shape of the preceding bubble.

(ii) When a pair of bubbles is formed not vertically in line, one bubble is immediately entrained into the wake of the other bubble and is realigned vertically.

(iii) The trailing bubble starts to rise, changing its shape and increasing velocity. The aspect ratio $h / d_{b}$ changes from 0.28 to around 0.7 . This indicates that the width and intensity of the wake vary with the distance from the bubble. When the trailing bubble catches up with the preceding bubble, they coalesce to form a larger bubble.

For other wake situations, the results of the observations are shown schematically in Fig. 5. In the range of $R e ; 90-250$, the trailing bubble follows a helixlike path. Although the wake extends a longer distance than the laminar wake, the approaching velocity is considerably reduced. This implies the existence of a less intensive wake. In the range of $R e ; 250-500$, the behavior becomes less reproducible. In some cases, the wake of the preceding bubble attracts the trailing bubble. In the case of a large trailing bubble of approximately critical size (about $d_{e}=2.1 \mathrm{~cm}$ in the liquid flow field), the wake often causes the trailing bubble to break up, and the resultant debris starts to rise to the preceding bubble.

In the range of $R e>500$, the shape of the bubbles becomes irregular and the preceding bubble $\left(d_{e}=\right.$ $1.7 \mathrm{~cm}$ ) itself often breaks up while preparing the trailing bubble. Owing to the unsystematic configuration of the wake and to the unstable bubble situation, the effect of the wake on the interaction between the bubbles is small, and the bubbles do not always collide. Even when they collide, they are liable to rupture immediately on collision.

2) Acceleration effect on the trailing bubble

The velocity ratios of the preceding and trailing bubbles are plotted against separation distance in

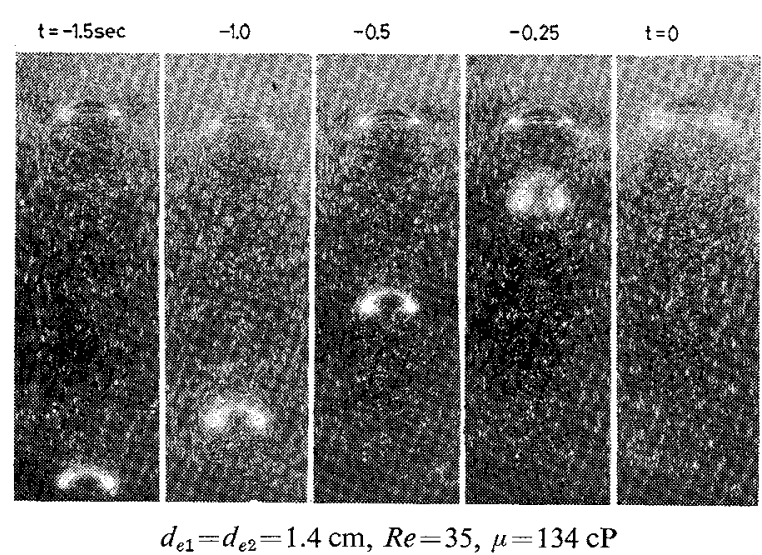

Fig. 8 Typical photographs of interaction of two bubbles with laminar wakes

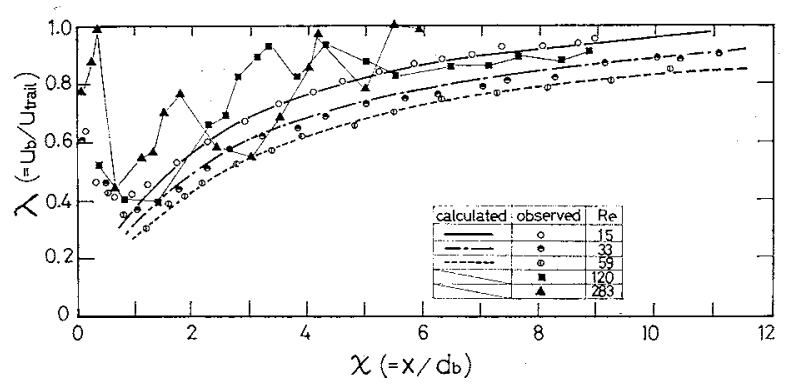

Fig. 9 Velocity ratio of two bubbles and comparison of observed and calculated values

Fig. 9 with Reynolds number as a parameter. For bubbles with transitional wakes $(R e=120,283)$, there exists a certain acceleration effect, since the values of the ratios are less than one. At a larger value of $R e=283$, a systematic variation of the ratios can no longer be seen, and the acceleration effect will expire at the short distance of $\chi=5-6$.

For bubbles with laminar wakes $(10<\operatorname{Re}<90)$, on the other hand, there exists a systematic variation in the velocity ratios with Reynolds number and separation distance. The ratio decreases with distance in the region of $0<\chi<1$, and then increases until it approaches an asymptote value of $\lambda=1$ far downstream from the preceding bubble. Comparing the results of the wake velocities shown in Fig. 7 with those of the velocity ratios of the bubbles shown in Fig. 9, the following description can be made of the acceleration effect. The trailing bubble, once being induced into the laminar wake of the preceding bubble, starts to rise with an additional unsteady velocity component. This additional component increases until the trailing bubble arrives at the point of a distance of a basal diameter from the bubble $(\chi=1)$ and then decreases until the bubble finally collides with the preceding bubble. At the final stage of travel of the trailing bubble $(0<\chi<1)$, an additional force is required to remove intervening liquid between the two bubbles, and the shape of the trailing bubble is deformed as shown in 


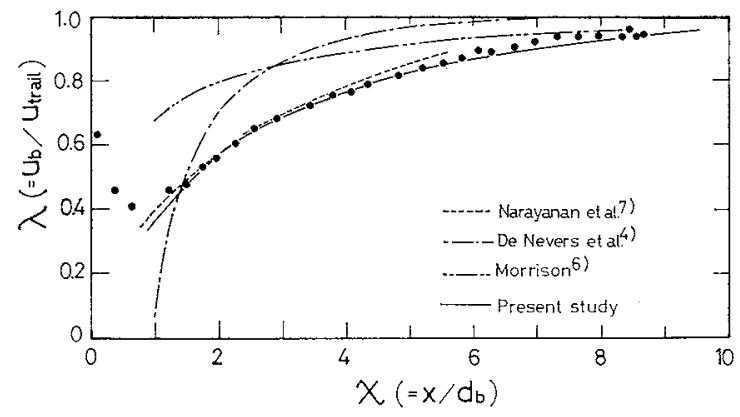

Fig. 10 Velocity ratio of two bubbles calculated according to several empirical equations

Fig. 8. The situation in this small region seems to be very complicated.

An attempt is made to find the relation existing between the additional velocity component of the trailing bubble and the intensity scale of the wake of the preceding bubble. The present treatment is restricted to bubbles with laminar wakes and for a distance of separation greater than the basal diameter. As a measure of the intensity scale, the wake velocity on the symmetric axis of the wake is adopted since the concept of wake volume cannot be applied to the effect still existing at a long distance downstream from the bubble. Two bubbles of equal size and equal terminal rising velocity are considered.

If the additional velocity component is assigned as $\gamma$, the velocity of the trailing bubble is

$$
u_{\mathrm{tra} \mathrm{i} 1}=u_{b}+\gamma
$$

Assuming that this component can be equated to the difference in velocities between the wake and the main liquid flow, that is,

$$
\gamma=u_{b}-u_{w \cdot a \times i s}
$$

the velocity of the trailing bubble is expressed as

$$
u_{\text {trai1 }}=2 u_{b}-u_{w \cdot a x i s}
$$

The velocity of the preceding bubble has been found not to be affected by the trailing bubble, and therefore the ratio of the two bubbles is expressed as

$$
\lambda\left(=u_{b} / u_{\mathrm{tra} 1}\right)=1 /\left(2-u_{w \cdot \mathrm{axis}} / u_{b}\right)
$$

Using Eq. (9),

$$
\lambda=1 /\left(2-1.3 R e^{-0.05}+0.85 R e^{0.27} / \chi\right)
$$

The ratios calculated according to Eq. (14) are shown in Fig. 9. The agreement between observed and calculated values is surprisingly good, in spite of the major assumption.

Using several published correlations for the acceleration effect, the velocity ratios are calculated for a value of $R e=15$ and shown in Fig. 10 in comparison with the present results. The predicted values of Narayanan et al..$^{7}$, although their correlation is valid only in the range of $\chi<5.5$, seem to be very close to the present results. Their correlation was obtained by direct measurement of the velocities of the two bubbles. Other workers used assumed wake models which are inapplicable to the whole range of the actual wake of spherical-cap bubbles.

\section{Conclusion}

1) No significant differences were observed in the dynamical behavior of single bubbles, for free-rising bubbles in a stationary liquid pool and for bubbles held stationary in a vertical water channel. The system of bubbles held stationary is considered to be acceptable for the study of the interaction process of a pair of spherical-cap bubbles.

2) The fluid dynamical properties of the wakes behind spherical-cap bubbles were classified by Reynolds number, as a laminar wake $(10<\operatorname{Re}<90)$, a transitional wake $(90<\operatorname{Re}<500)$, and a turbulent wake $(R e>500)$.

3) The laminar wake has a long, stable cylindrical tail. The trailing bubble, once being induced into the tail, starts to rise with an additional unsteady velocity component. When the trailing bubble catches up with the preceding one, they coalesce to form a larger one. Comparing the wake velocity and the additional velocity component of the trailing bubble, the additional component was found to be equated with the difference in velocities of main liquid flow and of the wake of the preceding bubble.

4) The turbulent wake has an unstable tail, which has a limited effect on the acceleration of the trailing bubble. Hence the two bubbles aligned vertically do not always collide, and even if they collide, they are liable to rupture.

\section{Acknowledgment}

The authors gratefully acknowledge the experimental assistance of Messrs. H. Imaizumi and T. Yamashita.

$$
\begin{aligned}
& \text { Nomenclature } \\
& d_{b} \quad=\text { basal diameter of spherical-cap bubble [cm] } \\
& d_{e} \quad=\text { equivalent diameter of spherical-cap bubble }[\mathrm{cm}] \\
& d_{w} \quad=\text { maximum diameter of wake } \\
& F_{r} \quad=\text { Froude number }\left(=u_{b} / \sqrt{g\left(d_{e} / 2\right)}\right) \\
& g \quad=\text { acceleration due to gravity }\left[\mathrm{cm} / \mathrm{sec}^{2}\right] \\
& h \quad=\text { height of spherical-cap bubble } \quad[\mathrm{cm}] \\
& l_{w} \quad=\text { distance between frontal surface of bubble } \\
& \text { and stagnant point in wake [cm] } \\
& M \quad=\text { Morton number }\left(=g \mu^{4} / \rho \lambda^{3}\right) \text { [-] } \\
& R \quad=\text { frontal radius of curvature of spherical-cap } \\
& \text { bubble } \\
& \text { [cm] } \\
& \text { Re } \quad=\text { Reynolds number }\left(=d_{e} u_{b} \rho / \mu\right) \text { [-] } \\
& u_{0}=\text { terminal rising velocity of bubble with } \\
& \text { respect to liquid }[\mathrm{cm} / \mathrm{sec}] \\
& u_{\text {trail }}=\text { unsteady velocity of trailing bubble } \quad[\mathrm{cm} / \mathrm{sec}] \\
& u_{w \cdot \operatorname{axis}}=\text { wake velocity on symmetrical axis of wake } \\
& V_{b} \quad=\text { bubble volume } \quad\left[\mathrm{cm}^{3}\right] \\
& V_{w} \quad=\text { wake volume } \quad\left[\mathrm{cm}^{3}\right] \\
& x=\text { separation distance of the two bubbles, or }
\end{aligned}
$$


separation distance from a bubble to a point

$=$ additional unsteady velocity component of trailing bubble

$[\mathrm{cm} / \mathrm{sec}]$

= maximum angle of spherical-cap bubble [deg]

$=$ liquid viscosity

[cP]

$=$ liquid density

$\left[\mathrm{g} / \mathrm{cm}^{3}\right]$

$=$ interfacial tension [dyne/cm]

$=$ velocity ratio of two bubbles $\left(=u_{b} / u_{\mathrm{trail}}\right)$

$=$ dimensionless separation distance $\left(=x / d_{b}\right)$

$=$ critical separation distance at which wake velocity is acquired
3) Davies, R. M. and G. Taylor: Proc. R. Soc. London, Ser. $A, \mathbf{A 2 0 0 ,} 375$ (1950).

4) De Nevers, N. and J. L. Wu: $A I C h E J ., 17,182$ (1971).

5) Kojima, E., T. Akehata and T. Shirai: J. Chem. Eng. Japan, 8, 108 (1975).

6) Morrison, F. A.: Chem. Eng. Sci., 28, 1115 (1973).

7) Narayanan, S., L. H. J. Goossens and N. W. F. Kossen: ibid., 29, 2071 (1974).

8) Otake, T., S. Tone, K. Nakao and Y. Mitsuhashi: ibid., 32, 377 (1977).

9) Parlange, J. Y.: J. Fluid Mech., 37, 257 (1964).

10) Tadaki, T. and S. Maeda: Kagaku Kōgaku, 25, 254 (1961).

11) Wegener, P. P. and J. Y. Parlange: Annual Review of Fluid Mechanics, 5, 79 (1973).

12) Yabe, I. and D. Kunii: Kogaku Kogaku Ronbunsyu, 2, 144 (1976).

(Presented in part at the 12th Autumnal Meeting of The Soc. of Chem. Engrs., Japan, Okayama, Oct. 9, 1978.) 